\title{
INTEGRAL and Swift observations of the supergiant fast X-ray transient AX J1845.0-0433 = IGR J18450-0435
}

\author{
V. Sguera ${ }^{1}$, A. J. Bird ${ }^{1}$, A. J. Dean ${ }^{1}$, A. Bazzano ${ }^{2}$, P. Ubertini' ${ }^{2}$, R. Landi ${ }^{3}$, A. Malizia ${ }^{3}$, E. J. Barlow ${ }^{1}$, \\ D. J. Clark ${ }^{1}$, A. B. Hill ${ }^{1}$, and M. Molina ${ }^{1}$ \\ 1 School of Physics and Astronomy, University of Southampton, Highfield SO17 1BJ, UK \\ e-mail: sguera@astro.soton.ac.uk \\ 2 IASF/CNR, via Fosso del Cavaliere 100, 00133 Roma, Italy \\ 3 IASF/CNR, via Piero Gobetti 101, 40129 Bologna, Italy
}

Received 8 August 2006 / Accepted 25 October 2006

ABSTRACT

\begin{abstract}
Context. AX J1845.0-0433 was discovered by ASCA in 1993 during fast outburst activity characterized by several flares on short timescales. Up to now, the source was not detected again by any X-ray mission. Its optical counterpart is suggested to be an O9.5I supergiant star, which is the only remarkable object found inside the ASCA error box.

Aims. To detect and characterize new fast outbursts of AX J1845.0-0433 and confirm its supergiant HMXB nature, using INTEGRAL and archival Swift XRT observations.

Methods. We performed an analysis of INTEGRAL IBIS and JEM-X data with OSA 5.1 as well as an analysis of archive Swift XRT data.

Results. We report on fast flaring activity from the source on timescales of a few tens of minutes, the first to be reported since its discovery in 1993. Two outbursts have been detected by INTEGRAL (Apr. 2005 and Apr. 2006) with similar peak fluxes and peak luminosities of $\sim 80 \mathrm{mCrab}$ and $9.3 \times 10^{35} \mathrm{erg} \mathrm{s}^{-1}(20-40 \mathrm{keV})$, respectively. Two other outbursts were detected by Swift XRT on Nov. 2005 and Mar. 2006. The refined Swift XRT position of AX J1845.0-0433 confirms its association with the supergiant star previously proposed as optical counterpart.

Conclusions. Our INTEGRAL and Swift results fully confirm the supergiant HMXB nature of AX J1845.0-0433 which can therefore be classified as a supergiant fast X-ray transient. Moreover they provide for the first time evidence of its recurrent fast transient behaviour.
\end{abstract}

Key words. gamma rays: observations $-\mathrm{X}$-rays: binaries $-\mathrm{X}$-rays: general

\section{Introduction}

The X-ray transient AX J1845.0-0433 was discovered in the Scutum arm region on 18 October 1993 during an ASCA observation lasting $\sim 16 \mathrm{~h}$ (Yamauchi et al. 1995). During the initial $\sim 9 \mathrm{~h}$, the source was in a very faint quiescent state with a $0.7-10 \mathrm{keV}$ flux of $\sim 3 \times 10^{-12} \mathrm{erg} \mathrm{cm}^{-2} \mathrm{~s}^{-1}$, then suddenly it flared up reaching a peak flux of $\sim 10^{-9} \mathrm{erg} \mathrm{cm}^{-2} \mathrm{~s}^{-1}$ in less than $20 \mathrm{~min}$. Subsequently the source was characterized by several other peaks lasting a few tens of minutes, until the end of the observation. Optical and infrared measurements of the ASCA error circle ( 1 arcmin radius) of AX J1845.0-0433 were performed by Coe et al. (1996). The only object of interest is an O9.5I supergiant star which was proposed by Coe et al. (1996) as the optical counterpart. Its estimated distance was $\sim 3.6 \mathrm{kpc}$, consistent with that derived from ASCA X-ray measurements. However Coe et al. (1996) pointed out that the error on the distance could be large, mainly because of the uncertainty in the reddening law. With these assumptions, the quiescent and the peak luminosity $(0.7-10 \mathrm{keV})$ of the source measured by ASCA were $4.6 \times 10^{33} \mathrm{erg} \mathrm{s}^{-1}$ and $1.5 \times 10^{36} \mathrm{erg} \mathrm{s}^{-1}$, respectively. The ASCA X-ray spectrum of the flare state was fitted by an absorbed power law with $\Gamma \sim 1$ and $N_{\mathrm{H}}=3.6 \pm 0.3 \times 10^{22} \mathrm{~cm}^{-2}$; no coherent pulsation was found in the range from $125 \mathrm{~ms}$ to $4096 \mathrm{~s}$ (Yamauchi et al. 1995).

Since the ASCA discovery, no further outburst activity has been reported from the source by any X-ray mission. The fast X-ray transient behaviour of AX J1845.0-0433 as well as its likely, but not entirely definitive, association with a supergiant star, suggest its classification as a possible member of the recently newly discovered class of Supergiant Fast X-ray Transients: SFXTs (Negueruela et al. 2005; Sguera et al. 2005, 2006). They are supergiant high mass X-ray binaries (SGXBs), which most of the time are undetectable having typical quiescence luminosities $\leq 10^{32}-10^{33} \mathrm{erg} \mathrm{s}^{-1}$. Occasionally they undergo fast X-ray transient activity lasting typically less than a day and characterized by several fast flares with timescales of a few tens of minutes and typical peak luminosities of $\sim 10^{36} \mathrm{erg} \mathrm{s}^{-1}$.

Recently, Halpern et al. (2006) noted that AX J1845.0-0433 is very likely the same object as IGR J18450-0435. Using the precise position of its optical counterpart, AX J1845.0-0433 is located 2!3 off the IBIS position of IGR J18450-0435 reported by Bird et al. (2006), slightly outside the ISGRI error circle. IGR J18450-0435 was firstly discovered by INTEGRAL during a survey of the Sagittarius arm tangent region in the spring 


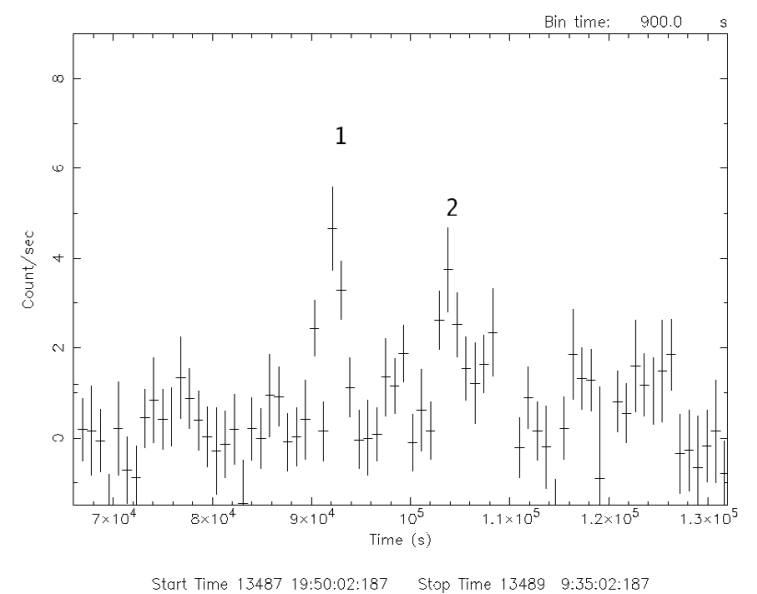

Fig. 1. ISGRI light curve (20-40 keV) of a newly discovered outburst of IGR J18450-0433 on 28 April 2005.

2003 (Molkov et al. 2004) at an average flux of $1.5 \pm 0.3 \mathrm{mCrab}$ (18-60 keV).

Here we report on the renewed fast outburst activity detected by INTEGRAL and Swift from AX J1845.0-0433, the first to be revealed since its discovery in 1993.

\section{INTEGRAL observations and results}

The INTEGRAL gamma-ray observatory carries three coaligned coded mask telescopes: the imager IBIS (Ubertini et al. 2003), the spectrometer SPI (Vedrenne et al. 2003) and the X-ray monitor JEM-X (Lund et al. 2003). In this work only results from IBIS/ISGRI (Lebrun et al. 2003) and JEM-X will be presented. The reduction and analysis of the IBIS/ISGRI and the JEM-X data have been performed using the INTEGRAL Offline Scientific Analysis (OSA) v.5.1. INTEGRAL observations are typically divided into short pointings (Science Window, $\mathrm{ScW}$ ) of $\sim 2000$ s duration.

We performed an analysis at the ScW level of the deconvolved ISGRI shadowgrams searching for outburst activity from IGR J18450-0435. In most of the ScWs, the source was well below a $5 \sigma$ detection level (20-40 keV), and only in two ScWs in revolution 310 and one $\mathrm{ScW}$ in revolution 429 was it significantly detected at $\sim 6 \sigma$ level $(20-40 \mathrm{keV})$. Our analysis showed that these detections corresponded to fast flares reaching their peak in a few tens of minutes and then dropping on the same timescale. In Sects. 2.1 and 2.2 we report on INTEGRAL results of each outburst.

\subsection{INTEGRAL results on the first outburst activity}

Figure 1 displays the 20-40 keV light curve of IGR J184500435. As we can see, the source underwent two fast flares (Nos. 1 and 2 in Fig. 1) with timescales of a few tens of minutes on 28 April 2005 at $\sim 19: 40$ UTC. The first flare reached a peak flux of $\sim 60 \mathrm{mCrab}(20-40 \mathrm{keV})$. Assuming a distance of $3.6 \mathrm{kpc}$, the $20-40 \mathrm{keV}$ peak luminosity was $\sim 7 \times 10^{35} \mathrm{erg} \mathrm{s}^{-1}$, in agreement with typical outburst luminosities of SFXTs (Sguera et al. 2006). After the second flare, the source seemed to show no further X-ray flaring activity. However the light curve is unfortunately truncated at the end because the source went outside the IBIS FOV. It is not possible to constrain the overall duration of the outburst activity and we cannot exclude the possibility that further flares took place. The INTEGRAL observation coverage

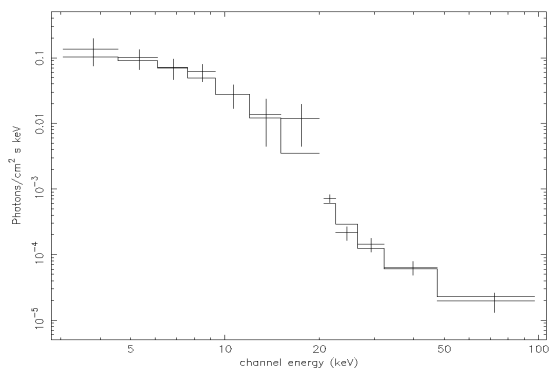

Fig. 2. Unfolded BMC broad band spectrum (3-100 keV) of AX J1845.0-0433 during the flare that occurred on 28 April 2005.

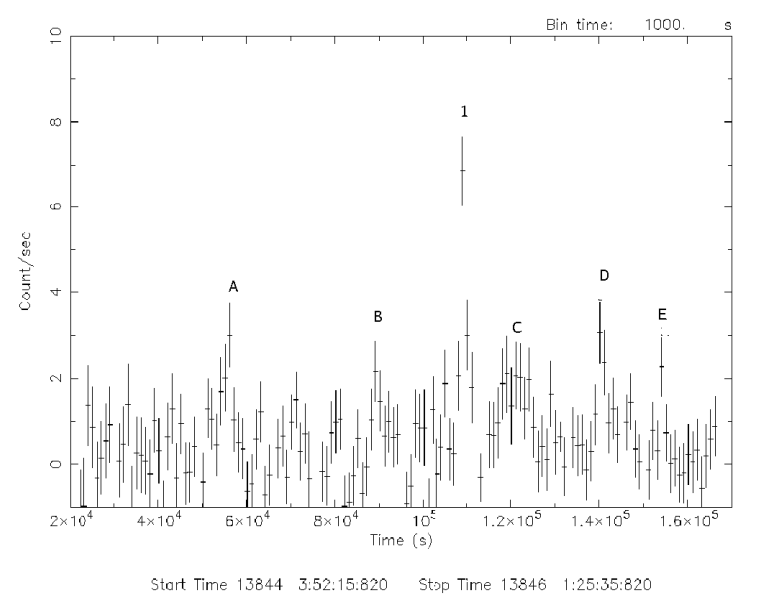

Fig. 3. ISGRI light curve (20-40 keV) of a newly discovered outburst of AX J1845.0-0433 which occurred on 20 April 2006.

since the source turned on up to the end of the light curve is $\sim 11 \mathrm{~h}$. A search for subsequent flaring activity, when the source was again in the IBIS FOV $\sim 1.4$ days later, did not give any positive results.

The first flare (No. 1 in Fig. 1) was also covered with JEM-X data. The $3-10 \mathrm{keV}$ peak flux and peak luminosity were $\sim 32 \mathrm{mCrab}$ and $\sim 7.4 \times 10^{35} \mathrm{erg} \mathrm{s}^{-1}$, respectively. The combined JEM-X and ISGRI spectrum (3-100 keV) during the first flare was reasonably fitted by a single power law $\left(\chi_{v}^{2}=\right.$ $1.12,156$ d.o.f.) with $\Gamma=2.5_{-0.5}^{+0.6}$. The fit improved adding a black body to the power law $\left(\chi_{y}^{2}=1.02,154\right.$ d.o.f. $)$, being the best fit parameters $k T=2_{-0.5}^{+0.7}$ and $\Gamma=1.7_{-0.8}^{+0.7}$. To account for a cross-calibration mismatch between the two instruments we have introduced a constant in the fit, which when left free to vary provides a value of $0.02_{-0.016}^{+0.08}$. An equally good fit $\left(\chi_{v}^{2}=1.02,154\right.$ d.o.f.) was also achieved using a Bulk Motion Comptonization model BMC (see Fig. 2).

Our analysis of this first flare enabled the determination of an ISGRI position for IGR J18450-0435 (RA = $18^{\mathrm{h}} 45^{\mathrm{m}} 03^{\mathrm{s}} .3 \mathrm{Dec}=-04^{\circ} 34^{\prime} 05^{\prime \prime} \cdot 5,(\mathrm{~J} 2000)$, error radius of 2.4$)$ which is located 0.5 from the optical counterpart of AX J1845.0-0433. This fully confirms that AX J1845.0-0433 and IGR J18450-0435 are indeed the same source.

\subsection{INTEGRAL results on the second outburst activity}

Figure 3 displays the 20-40 keV light curve of IGR J184500435 using recent Core Program data in revolution 429. As we can note, it strongly resembles the light curve of the previous flaring activity reported in Sect. 2.1. Most of the time the source did not show any relevant outburst activity, with a count rate less 


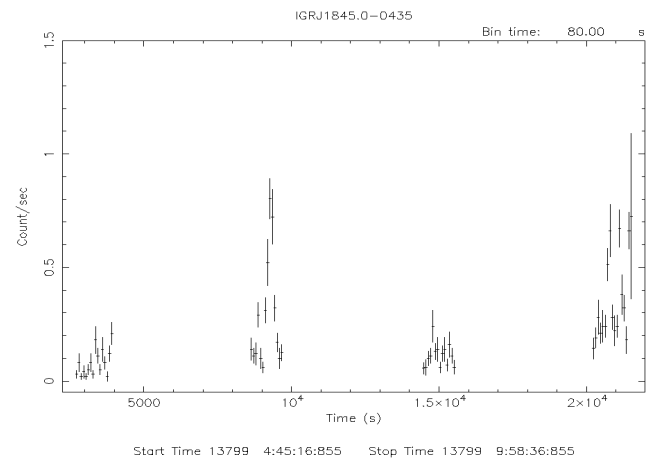

Fig. 4. Swift XRT light curve of AX J1845.0-0433 (0.2-10 keV) during the observation on 5 March 2006.

than 1 count/s, however several very quick flares (labeled from A to E in Fig. 3) appeared on a timescale of tens of minutes. Their peak flux never reached a value greater than $\sim 30 \mathrm{mCrab}(20-$ $40 \mathrm{keV}$ ). Moreover a noticeable very fast and strong flare (No. 1 in Fig. 3) occurred on 9:30 UTC 20 April 2006. It reached a peak flux of $\sim 80 \mathrm{mCrab}$ or $6 \times 10^{-10} \mathrm{erg} \mathrm{cm}^{-2} \mathrm{~s}^{-1}(20-40 \mathrm{keV})$ in $\sim 30 \mathrm{~min}$ and then it dropped with the same timescale. The $20-40 \mathrm{keV}$ peak luminosity was $\sim 9.3 \times 10^{35} \mathrm{erg} \mathrm{s}^{-1}$. These values are slightly greater than the previous ones relating to the flaring activity detected by INTEGRAL about one year before (Sect. 2.1). The light curve in Fig. 3, pertaining to rev 429, is truncated at the beginning and at the end. A search for more flaring activity before and after this revolution did not give any results. We extracted an ISGRI spectrum during this fast flare $(20-100 \mathrm{keV})$, the best fit $\left(\chi_{v}^{2}=1.18,24\right.$ d.o.f.) was provided applying a single power law with $\Gamma=2.9_{-0.7}^{+0.9}$. However a BMC model also provided a good description to the data $\left(\chi_{v}^{2}=\right.$ 1.2, 22 d.o.f.)

\section{Swift observations and results}

In this section we present $\mathrm{X}$-ray observations acquired with the XRT (X-ray Telescope, $0.2-10 \mathrm{keV}$ ) on board the Swift satellite (Gehrels et al. 2004). A search of the XRT data archive revealed that Swift carried out 2 observations of IGR J18450-0433 on 11 November 2005 and 5 March 2006. Unfortunately in both observations the source was outside the IBIS FOV so we had no simultaneous ISGRI data. The XRT collected data for a total exposure time of $4.6 \mathrm{ks}$ and $4.8 \mathrm{ks}$, respectively. The data reduction was performed using XRTDAS v. 2.4 standard data pipeline package (XRTPIPELINE v. 0.10.3), in order to produce screened event files. All data were extracted only in the Photon Counting (PC) mode, adopting the standard grade filtering (0-12 for PC) according to the XRT nomenclature. Events for spectral analysis were extracted within a circular region of radius $20^{\prime \prime}$, which encloses about $90 \%$ of the PSF at $1.5 \mathrm{keV}$ (Moretti et al. 2004) centered on the source position. The background was extracted from various source-free regions close to the X-ray source of interest using both circular/annular regions with different radii, in order to ensure an evenly sampled background.

Figures 4 and 5 show the $0.2-10 \mathrm{keV}$ XRT light curve of IGR J18450-0433 during the observations performed on 5 March 2006 and 11 November 2005, respectively. We note that the temporal coverage was not continuous, there were significant gaps in both the light curves due to visibility constraints. Nevertheless the fast flaring behaviour of the source was evident, especially during the observation performed on 5 March 2006 (Fig. 4). On this occasion, initially the source displayed a very

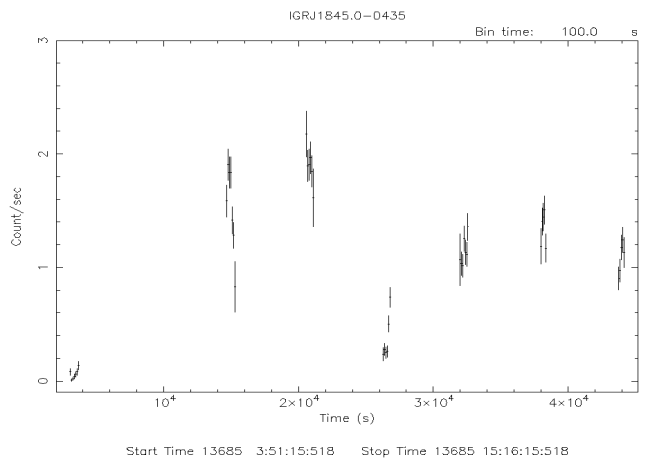

Fig. 5. Swift XRT light curve of AX J1845.0-0433 (0.2-10 keV) during the observation on 11 November 2005.

low count rate. Subsequently, it underwent a very fast flare reaching in only 4 min a peak flux and a peak luminosity of $\sim 1.1 \times 10^{-10} \mathrm{erg} \mathrm{cm}^{-2} \mathrm{~s}^{-1}$ and $\sim 2 \times 10^{35} \mathrm{erg} \mathrm{s}^{-1}$, respectively $(0.2-10 \mathrm{keV})$. Then the flux dropped again to a very low level with the same fast timescale. The spectrum $(0.2-8 \mathrm{keV})$ extracted during the observation 5 March 2006 was well fitted by an absorbed power law $\left(\chi_{v}^{2}=0.63,41\right.$ d.o.f $)$ with $\Gamma=$ $0.85 \pm 0.3$ and $N_{\mathrm{H}}=2.3 \pm 0.7 \times 10^{22} \mathrm{~cm}^{-2}$. However a good fit was also provided by a black body model $\left(\chi_{v}^{2}=0.7,42\right.$ d.o.f. $)$ with $k T=2.4 \pm 0.2$. The average $0.2-8 \mathrm{keV}$ flux was $\sim 2 \times$ $10^{-11} \mathrm{erg} \mathrm{cm}^{-2} \mathrm{~s}^{-1}$.

In the light curve in Fig. 5 (11 November 2005), initially the source was characterized by a count rate less than 0.1 count/s, then it flared up quickly reaching a peak flux and peak luminosity of $\sim 2.3 \times 10^{-10} \mathrm{erg} \mathrm{cm}^{-2} \mathrm{~s}^{-1}$ and $\sim 3.6 \times 10^{35} \mathrm{erg} \mathrm{s}^{-1}$ respectively $(0.2-10 \mathrm{keV})$. The spectrum $(0.2-10 \mathrm{keV})$ was best fitted by an absorbed power law $\left(\chi_{v}^{2}=1.28,166\right.$ d.o.f.) with $\Gamma=0.75 \pm$ 0.1 and $N_{\mathrm{H}}=1.6 \pm 0.18 \times 10^{22} \mathrm{~cm}^{-2}$. The latter is in agreement with the galactic absorption along the line of sight $\left(N_{\mathrm{H}}=\right.$ $\left.1.58 \times 10^{22} \mathrm{~cm}^{-2}\right)$. A simple power law or thermal models, such as blackbody or bremsstrahlung, instead provided very poor fits. The $0.2-10 \mathrm{keV}$ average flux was $\sim 1.3 \times 10^{-10} \mathrm{erg} \mathrm{cm}^{-2} \mathrm{~s}^{-1}$, which is an order of magnitude higher than that during the other observation.

Our Swift analysis provided a very accurate position of AX J1845.0-0433 (RA $=18^{\mathrm{h}} 45^{\mathrm{m}} 01^{\mathrm{s}} .9$ Dec $\left.=-04^{\circ} 33^{\prime} 57^{\prime \prime} .6\right)$ which is located $4^{\prime \prime} .7$ from the supergiant star proposed by Coe et al. (1996) as its optical counterpart. This fully confirms the supergiant HMXB nature of AX J1845.0-0433.

\section{Conclusions}

To date, the only X-ray detection of AX J1845.0-0433 in outburst dates back to 1993 . No X-ray mission provided a regular monitoring of the Scutum arm region, which is essential to detect fast X-ray transient activity from a source like AX J1845.0-0433. The INTEGRAL satellite is regularly monitoring the Scutum arm region performing cycles of observations with an exposure time that is $\sim 10$ days. We reported on renewed fast outburst activity from AX J1845.0-0433 discovered by INTEGRAL and also on results of two Swift observations from archival data. Table 1 provides a summary of the characteristics of all outbursts from AX J1845.0-0433 detected to date.

As for the newly discovered outbursts by INTEGRAL, they occurred on 28 April 2005 and 20 April 2006. The strongest one was characterized by a $20-40 \mathrm{keV}$ peak flux and peak luminosity of $\sim 80 \mathrm{mCrab}\left(6 \times 10^{-10} \mathrm{erg} \mathrm{cm}^{-2} \mathrm{~s}^{-1}\right)$ and $\sim 9 \times 10^{35} \mathrm{erg} \mathrm{s}^{-1}$, respectively. In both of them, the source displayed a kind of 
Table 1. Summary of INTEGRAL, Swift and ASCA observations of outbursts of AX J1845.0-0433.

\begin{tabular}{cccccccc}
\hline \hline No. & Observation & Date & $\begin{array}{c}\text { Energy band } \\
(\mathrm{keV})\end{array}$ & $\begin{array}{c}\text { Peak flux } \\
\left(\mathrm{erg} \mathrm{cm}^{-2} \mathrm{~s}^{-1}\right)\end{array}$ & $\begin{array}{c}\text { Peak luminosity } \\
\left(\mathrm{erg} \mathrm{s}^{-1}\right)\end{array}$ & $\begin{array}{c}\text { Photon index } \\
(\text { power law })\end{array}$ & $\begin{array}{c}N_{\mathrm{H}} \\
\left(\mathrm{cm}^{-2}\right)\end{array}$ \\
\hline 1 & ASCA & 18 Oct. 1993 & $0.7-10$ & $1 \times 10^{-9}$ & $1.5 \times 10^{36}$ & $1_{-0.07}^{+0.07}$ & $3.6 \pm 0.3 \times 10^{22}$ \\
2 & INTEGRAL/ISGRI & 28 Apr. 2005 & $20-40$ & $4.5 \times 10^{-10}$ & $7 \times 10^{35}$ & $2.5_{-0.5}^{+0.6}$ & \\
3 & INTEGRAL/JEM-X & 28 Apr. 2005 & $3-10$ & $4.8 \times 10^{-10}$ & $7.4 \times 10^{35}$ & & \\
4 & Swift XRT & 11 Nov. 2005 & $0.2-10$ & $2.3 \times 10^{-10}$ & $3.6 \times 10^{35}$ & $0.75_{-0.1}^{+0.1}$ & $1.6 \pm 0.18 \times 10^{22}$ \\
5 & Swift XRT & 5 Mar. 2006 & $0.2-10$ & $1.1 \times 10^{-10}$ & $2 \times 10^{35}$ & $0.85_{-0.3}^{+0.3}$ & $2.3 \pm 0.7 \times 10^{22}$ \\
6 & INTEGRAL/ISGRI & 20 Apr. 2006 & $20-40$ & $6 \times 10^{-10}$ & $9.3 \times 10^{35}$ & $2.9_{-0.7}^{+0.9}$ & \\
\hline
\end{tabular}

pre and post flaring activity characterized by several other small flares having the same fast timescale but a smaller peak flux which never reached a value greater than $30 \mathrm{mCrab}(20-40 \mathrm{keV})$. The combined JEM-X/ISGRI spectrum (3-100 keV) was well described by a power law plus black body model or by a Comptonized model (BMC).

From Swift XRT data analysis, we fully confirmed that the supergiant star O9.5I is the optical counterpart of AX J1845.0-0433 as result of the very accurate Swift position of AX J1845.0-0433, located 4.'7 from the star. During the first Swift observation (11 November 2005), the average flux of the source $\left(\sim 1.3 \times 10^{-10} \mathrm{erg} \mathrm{cm}^{-2} \mathrm{~s}^{-1}\right)$ was an order of magnitude higher than that during the second observation (5 March 2006). On the contrary, the spectrum was very similar in both observations being well fitted by an absorbed power law with $\Gamma \sim$ $0.75-0.85$ and $N_{\mathrm{H}} \sim 2 \times 10^{22} \mathrm{~cm}^{-2}$. The latter is similar to that measured by ASCA in 1993 (see Table 1). We point out that the galactic absorption along the line of sight is $1.58 \times 10^{22} \mathrm{~cm}^{-2}$.

The four newly discovered fast outbursts reported in this paper provided evidence of recurrent fast X-ray transient behaviour of the supergiant high mass X-ray binary AX J1845.0-0433. Its fast flaring activity sets it apart from classical SGXBs and allows its classification as member of the recently discovered class of Supergiant Fast X-ray Transients: SFXTs (Negueruela et al. 2005; Sguera et al. 2005, 2006). To date, AX J1845.0-0433 is the sixth such source to be detected by INTEGRAL. Its flaring behaviour is very similar to that of the others 5 SFXTs, with the same fast timescale, peak-flux and peak-luminosity. Fast
X-ray activity has been detected from the source in ASCA, Swift and INTEGRAL observations lasting only a few hours. Since INTEGRAL is regularly monitoring the Scutum arm region, further IBIS detections of AX J1845.0-0433 in outburst are not unexpected. This could allow us to search for recurrence time and in turn further insights into the system geometry.

Acknowledgements. We thank the anonymous referee for very useful comments which helped us to improve the paper. This research has been supported by University of Southampton School of Physics and Astronomy. AB, PU, AM, RL acknowledge the ASI financial support via grant I/R/046/04.

\section{References}

Bird, A. J., Barlow, E. J., Bassani, L., et al. 2006, ApJ, 636, 765 Coe, M. J., Fabregat, J., Negueruela, I, et al. 1996, MNRAS, 281, 333 Gehrels, N., Chincarini, G., Giommi, P., et al. 2004, ApJ, 611, 1005 Halpern, J. P., \& Gotthelf, E. V. 2006, ATEL, 692

Lebrun, F., Leray, J. P., Lavocat, P., et al. 2003, A\&A, 411, L141

Lund, N., Butz-Jorgentsen, C., Westergaard, N. J., et al. 2003, A\&A, 411, L231 Molkov, S. V., Cherepashchuk, A. M., Lutovinov, A. A., et al. 2004, A\&A, 30, L534

Moretti, A., Campana, S., Tagliaferri, G., et al. 2004, SPIE, 5165, 232 Negueruela, I., Smith, D. M., Reig, P., et al. 2005, ESA SP-604, 165 Sguera, V., Barlow, E. J., Bird, A. J., et al. 2005, A\&A, 444, 221

Sguera, V., Bazzano, A., Bird, A. J., et al. 2006, ApJ, 646, 452 Sunyaev, R. A., \& Titarchuk, L. 1980, A\&A, 86, 121

Ubertini, P., Lebrun, F., Di Cocco, G., et al. 2003, A\&A, 411, L131

Vedrenne, G., Roques, J., Schnfelder, V., et al. 2003, A\&A, 411, L63

Yamauchi, S., Aoki, T., Hayashida, K., et al. 1995, PASJ, 47, 189 